\title{
Broadband Purcell enhancement in plasmonic ring cavities
}

\author{
Ernst Jan R. Vesseur, ${ }^{1, *}$ F. Javier García de Abajo, ${ }^{2}$ and Albert Polman ${ }^{1}$ \\ ${ }^{1}$ Center for Nanophotonics, FOM Institute for Atomic and Molecular Physics (AMOLF), \\ Science Park 104, 1098 XG Amsterdam, The Netherlands \\ ${ }^{2}$ Instituto de Óptica, CSIC, Serrano 121, 28006 Madrid, Spain
}

(Received 27 May 2010; published 11 October 2010)

\begin{abstract}
We present a theoretical study of a surface plasmon ring resonator geometry that shows strong spontaneous emission control due to an extremely small optical cavity mode volume. $\mathrm{V}$ grooves made in the surface of a metal confine whispering gallery surface plasmon polariton modes at the bottom of the groove, with the confinement determined by groove depth and width. Resonances in ringlike cavities defined by grooves patterned into circular shape, determined using the boundary-element method, are in agreement with calculations based on dispersion for linear $\mathrm{V}$ grooves. Cavity quality factors are relatively insensitive to cavity geometry $(Q=10-50)$ while mode volume is very sensitive to small differences in the cavity shape. The smallest mode volume $V=0.00073 \lambda_{0}^{3}$ is found for a ring with a 10-nm wide, $100-\mathrm{nm}$ deep groove with straight sidewalls and a diameter of $180 \mathrm{~nm}$. Purcell factors well above 2000 are found in the energy range from $E$ $=1.0-1.8 \mathrm{eV}$ depending on cavity geometry. For a given cavity geometry the Purcell enhancement is observed over a broad spectral range (50-100 meV), enabling application of these cavities beyond the typical lowtemperature cavity quantum electrodynamics experiments.
\end{abstract}

DOI: 10.1103/PhysRevB.82.165419

PACS number(s): 73.20.Mf, 42.60.Da, 42.50.Pq

\section{INTRODUCTION}

Control over spontaneous emission is key to many photonic applications and also a subject of great fundamental interest. According to Fermi's golden rule, the rate of spontaneous emission of an optical emitter is proportional to the local density of optical states (LDOS). In a homogeneous medium, the spontaneous emission rate of an emitter is fixed and it depends on the transition dipole moment of the emitter and the refractive index of the surrounding medium. In an environment that is structured at the scale of the wavelength the LDOS can be spatially controlled. ${ }^{1}$ As a result, the spontaneous emission rate of an emitter can be locally increased or decreased, depending on the geometry.

An optical microcavity provides an environment in which the LDOS can be strongly increased. The increase in spontaneous emission in the cavity, known as the Purcell effect, ${ }^{2}$ is given by the Purcell factor,

$$
F_{P}=\frac{3}{4 \pi^{2}} \frac{Q}{V}\left(\frac{\lambda}{n}\right)^{3},
$$

where $Q$ is the cavity quality factor, which is proportional to the time light is stored in the cavity, and $V$ the mode volume, a measure for the spatial extent of the modal field inside the cavity; $n$ is the refractive index of the cavity medium.

Optimization of the Purcell factor enables low-threshold lasing, bright luminescent sources, and the possibility to avoid quenching in materials that classically show strong nonradiative decay. Most work so far has focused on achieving high Purcell factors by optimizing $Q$, for example in Fabry-Perot, microsphere, ${ }^{3,4}$ micropillar, ${ }^{5,6}$ microtoroid, ${ }^{7}$ microdisk, ${ }^{8}$ or photonic crystal cavities. ${ }^{9,10}$ The highest $Q$ factors were reached in microspheres $\left(Q=10^{10}\right)^{3}$; in integrated on-chip microcavities a $Q$ of $10^{7}$ was observed. ${ }^{7}$

One problem with using these high- $Q$ cavities is that due to the narrow linewidth associated with the high cavity $Q$, the interaction between light and matter occurs only over a very narrow bandwidth. Most emitters, however, have emission spectra with a broad linewidth. As a result, the coupling of these emitters to the cavities is relatively weak. The highest decay rate enhancements reported so far were measured on photonic crystal cavities doped with semiconductor quantum dots (QDs) ${ }_{1}^{11}$ for which a 75 -fold increase in decay rate at a wavelength of $945 \mathrm{~nm}$ was found. Moreover, such enhancements were only found at low temperature. ${ }^{5,11}$

An alternative way to increase the Purcell factor is to reduce the mode volume. If this can be achieved, high Purcell factors can be observed at modest $Q$ and thus over a broader bandwidth, enabling broadband light sources with high emission rate at room temperature.

So far, the smallest mode volumes are observed in photonic crystals, ${ }^{12}$ in which the mode volume $V$ is limited by diffraction; typically $V=(\lambda / n)^{3} \approx 0.1 \mu \mathrm{m}^{3}$. Much smaller mode volumes can be achieved using surface plasmon polaritons (SPPs), surface waves at a metal-dielectric interface resulting from the coupling between photons and the free electron plasma of the metal. SPPs are strongly bound to the interface and evanescently decay into either medium. The field confinement offered by plasmons allows enhanced interaction with emitters even for propagating modes. ${ }^{13,14}$

Three-dimensional (3D) confinement of plasmons was demonstrated in Fabry-Perot-type plasmon cavities where SPPs propagate either on a single metal interface or in the dielectric gap in a metal-insulator-metal (MIM) geometry, ${ }^{15-17}$ leading to a mode volume of $V=0.01(\lambda / n)^{3}$. Most recently, 3D MIM cavities were demonstrated having a smallest (predicted) volume $V=0.00033(\lambda / n)^{3}$ for a $105 \mathrm{~nm}$ diameter MIM disk. ${ }^{18}$

Indeed, MIM waveguides offer the smallest possible confinement of light, thus enabling the largest Purcell factor. So far, however, MIM cavities are made of closed material layers and are thus not easily accessible for optical probes of the LDOS. An MIM plasmon geometry that has a more open 
(a)

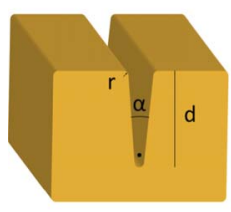

(b)

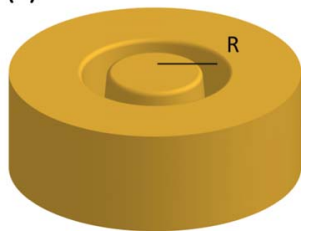

FIG. 1. (Color online) The boundary geometry as used in the calculations. (a) Groove parametrized by minor radius $r$, opening angle $\alpha$, and depth $d$, as used in 2D-BEM calculations. The solid dot indicates the position at which the local density of states was calculated. (b) Ring resonator based on the groove of (a), having major radius $R$, used in axially symmetric BEM calculations. The minor radius $r$ is used for smoothing the external edges and the bottom wedge of the groove.

geometry is a $\mathrm{V}$ groove made in the surface of a metal. ${ }^{19}$ Such V grooves provide strong confinement of light in the lateral dimension and if made into a ring they form a $3 \mathrm{D}$ plasmon cavity. In contrast to the planar MIM cavities demonstrated so far, these $\mathrm{V}$ grooves can be easily infilled with optical emitters or gain media.

Ring resonators based on V-groove plasmon waveguides $^{20-22}$ support whispering-gallery resonances due to circulating plasmons even when the circumference fits only a single plasmon wavelength, as we have shown previously. ${ }^{23}$ The resonances are easily tunable through both the diameter of the ring and the shape of the groove and they have an extremely small mode volume. The groove void allows for incorporation of emitters near the maximum of the electric field. Ring resonators based on surface plasmons are therefore a promising candidate for use as high $F_{P}$ cavities.

In this paper we investigate these V-groove ring cavities with the aim of determining the smallest possible mode volume while maintaining a reasonable cavity $Q$. We find a mode volume as small as $0.00073 \lambda_{0}^{3}$ at a cavity $Q=10-50$. The corresponding Purcell factor is $F_{P}>2000$. This opens up possibilities for broadband enhanced interaction between an emitter and light stored in the ring cavity.

\section{METHODS}

We have performed boundary-element-method (BEM) calculations ${ }^{24,25}$ in which Maxwell's equations are rigorously solved for an electric field that is expressed in terms of charges and currents on the metal surface. We first use a two-dimensional (2D) boundary [as shown in Fig. 1(a)] to calculate the optical properties of an infinitely long groove in an otherwise planar Au surface. In the direction along the groove $(z)$, the fields are taken to evolve as $e^{i k_{z} z}$. A similar groove geometry was then used to define a ring cavity [Fig. 1(b)]. Here, solutions for the field depend on azimuthal angle $\phi$ as $e^{i m \phi}$, with $m=0, \pm 1, \pm 2, \ldots$ In the calculation, contributions up to an order $|m|<5$ were taken into account, high enough for the results to reach convergence. The calculations are performed using tabulated optical constants for Au. ${ }^{26}$ The ring resonator is parametrized by four parameters: major radius $R$, groove depth $d$, opening angle of the groove $\alpha$, and minor radius $r=5 \mathrm{~nm}$ that describes the curvature of the cor-

ners and bottom of the groove. In the calculations we have varied $\alpha$ from $0^{\circ}$ to $10^{\circ}, d$ from 100 to $1000 \mathrm{~nm}$, and $R$ from 50 to $600 \mathrm{~nm}$. These parameters are at the limits of state-ofthe-art fabrication techniques such as focused-ion-beam milling. Recent advances ${ }^{23,27,28}$ show that grooves can be made narrower and narrower and that the ultimate limits of fabrication have not been reached. The choice of $\mathrm{Au}$ is motivated by existing experimental measurements of the resonance spectra of ring resonators on this material. ${ }^{23}$

\section{RESULTS}

\section{A. Dispersion in straight grooves}

Before treating resonant cavities, we first present the calculated dispersion relation and mode profiles for linear $\mathrm{V}$ grooves. Using BEM we calculate the LDOS, associated with the electric field, at a point inside the groove, at $10 \mathrm{~nm}$ above the bottom [solid dot in Fig. 1(a)]. The LDOS is calculated for a range of energies and separated into contributions arising from different wave vectors $k_{z}$ along the groove. ${ }^{29}$ Optical modes of the geometry show up as maxima in the LDOS. For each energy, we find the $k_{z}$ for which the LDOS peaks and so obtain the dispersion relation in this geometry. Figure 2(a) shows the dispersion of the three lowest-order plasmon modes for grooves with $d=500 \mathrm{~nm}$ and $\alpha=10^{\circ}$. Also plotted is the light line in vacuum. The dispersion of the lowest-order plasmon mode $(n=1)$ deviates significantly from the light line for energies as low as $1.1 \mathrm{eV}$, in contrast to what is observed for planar SPPs. As a result, the $n=1$ groove mode does not couple to light or planar SPPs. Similar high dispersion has also been found for plasmons in planar MIM slabs. ${ }^{30,31}$ The groove also supports higher order modes, with mode number $n$ defined by the number of field antinodes. The insets of Fig. 2(a) show the distribution of the electric field intensity for the $n=1,2$ modes. Both modes are confined well inside the $\mathrm{V}$ groove with the $n=1$ mode concentrated in the bottom of the $\mathrm{V}$ groove. This demonstrates how these linear $\mathrm{V}$ grooves offer strong 2D confinement. The effective index of a planar MIM-plasmon is strongly dependent on the separation between the two metal interfaces; it increases for smaller separation. $^{30}$ The strong confinement to the groove can be understood from the fact that the plasmon is concentrated in the region with the highest effective index, which is near the bottom of the groove, where the metal walls are closest together. ${ }^{32}$ Figure 2(a) shows that the $n=2$ and $n=3$ modes have a threshold within the energy range of the graph. At the threshold energy, the groove mode has no wave vector in the groove direction and is thus a completely delocalized plasmon along the groove. ${ }^{17}$

The dispersion of groove plasmons can be further increased by narrowing the groove, as is shown in Fig. 2(b), which shows the dispersion of the groove plasmon in a $d$ $=100 \mathrm{~nm}, \alpha=0^{\circ}, 10-\mathrm{nm}$ wide groove, in which the vertical walls are parallel. ${ }^{32}$ For example, at $2.0 \mathrm{eV}, k_{z}$ is three times larger than it is for free space light. As is shown in the inset in Fig. 2(b), the plasmon field is very well confined inside the narrow groove. The field of this mode at $1.8 \mathrm{eV}$ is plotted at the same length scale as in Fig. 2(a), demonstrating the 


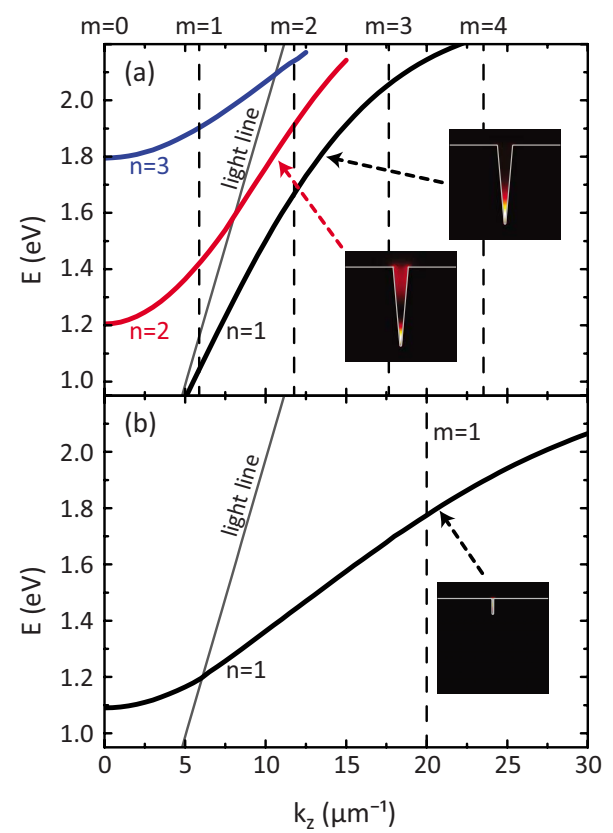

FIG. 2. (Color online) Dispersion of plasmons propagating in straight grooves in $\mathrm{Au}$ derived from BEM calculations of the LDOS. (a) V-grooves with $d=500 \mathrm{~nm}$ and $\alpha=10^{\circ}$. The lowestorder groove mode $(n=1)$ is below the light line in this energy range, so that it does not couple to light or planar SPPs. Higher orders have smaller $k_{z}$ and experience cutoff. For the two lowest orders, the electric field intensity is plotted at $1.8 \mathrm{eV}$. The $n=2$ mode has an extra node in its field distribution. (b) Dispersion for a $d=100 \mathrm{~nm}, \alpha=0^{\circ}$ (width $10 \mathrm{~nm}$ ) groove with the field intensity for the $n=1$ mode at $1.8 \mathrm{eV}$. Vertical dashed lines in both plots indicate the condition $k_{z}=m / R$ required for a resonance in a ring with radius (a) $R=170 \mathrm{~nm}$ and (b) $R=50 \mathrm{~nm}$, respectively. Ring resonances are expected at the intersection of the plasmon dispersion with these lines.

very high degree of confinement possible using V-groove plasmons. In this groove with parallel sidewalls, the field is not confined to the bottom but there is still strong vertical confinement due to the small depth of the groove $(d$ $=100 \mathrm{~nm}$ ). These shallow grooves show modal cutoff at $\sim 1.1 \mathrm{eV}$.

\section{B. Ring resonances}

Next, we study plasmons confined in V-groove ring cavities. Resonant circulating groove plasmons satisfy the relation $k_{z}=m / R$, where $k_{z}$ is the wave vector of the straightgroove plasmon, $m$ is the azimuthal order, i.e., the number of wavelengths that equals a circumference, and $R$ is the ring radius. We can now find the resonant energies for a ring with radius $R$ using the calculated dispersion relation. Dashed lines in Fig. 2(a) indicate the values of $k_{z}$ for resonances in an $R=170 \mathrm{~nm}$ ring. At the energies of intersection of a groove mode with a dashed line, the resonance condition is satisfied. We verify that these resonances indeed exist by calculating the LDOS spectrum. Figure 3(a) shows the BEMcalculated LDOS, normalized to the vacuum LDOS, in an $R=170 \mathrm{~nm}$ ring with a $d=500 \mathrm{~nm}, \alpha=10^{\circ}$ groove corre-

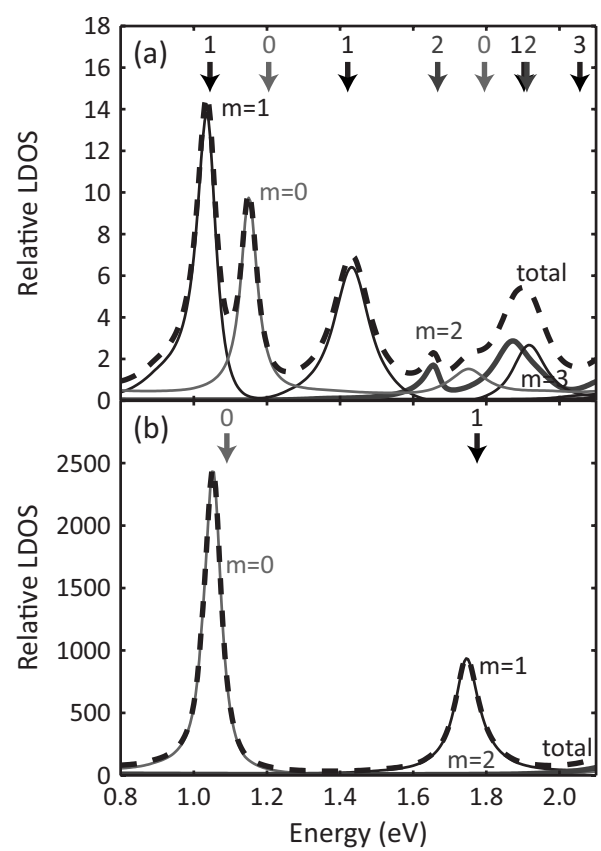

FIG. 3. Photonic local density of states (dashed lines) in the groove of a ring resonator at $10 \mathrm{~nm}$ above the groove bottom. (a) Ring with $d=500 \mathrm{~nm}, \alpha=10^{\circ}$, and $R=170 \mathrm{~nm}$. (b) Shallower, smaller ring with $d=100 \mathrm{~nm}, \alpha=0^{\circ}$, and $R=50 \mathrm{~nm}$. The LDOS, which is normalized to the vacuum LDOS, peaks at the resonance energies. Separate azimuthal mode $(m)$ contributions are plotted by the solid curves, following from the $3 \mathrm{D}$ calculation. For comparison, arrows indicate the resonant energies derived from the straightgroove dispersion (Fig. 2) with the corresponding $m$. Even for small ring radius the model predicts the resonances reasonably well.

sponding to the straight groove of Fig. 2(a). The peaks in the spectrum (dashed curve) represent optical resonances of the geometry. The separate azimuthal contributions to the LDOS are indicated by the drawn curves, with the corresponding $m$ values indicated next to the curves. The arrows drawn near the top horizontal axis of Fig. 3(a) indicate ring resonance energies derived from Fig. 2(a); the numbers denote the corresponding azimuthal order $m$. Both the resonant energies and the mode numbers agree well.

Figure 3(b) shows the LDOS spectrum of an $R=50 \mathrm{~nm}$ ring with a $d=100 \mathrm{~nm}, \alpha=0^{\circ}$ groove, corresponding to the geometry of Fig. 2(b). Due to the small radius, there are fewer resonances over the spectral range in Fig. 3. Interestingly, the LDOS reaches values well over 2000, demonstrating the potential of these rings for strong spontaneous emission control. Analogous to panel (a), the arrows in Fig. 3(b) indicate resonances derived from the straight-groove dispersion of Fig. 2(b). Again, good agreement is observed.

Figure 3 shows that plasmonic ring resonances, even in resonators with single-wavelength circumference $(m=1)$, directly follow from the straight groove plasmon dispersion. The small deviations between the energies indicated by the arrows and the actual resonances in the LDOS are attributed to interaction of the V-groove modes over the center plateau, ${ }^{23}$ which is not included in the simple straight-groove model. 


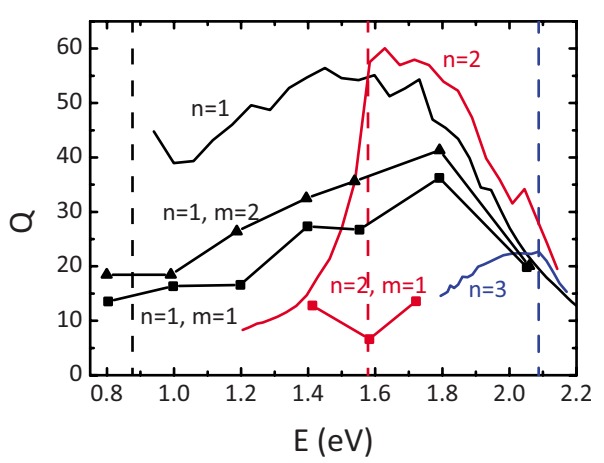

FIG. 4. (Color online) Quality factors derived from LDOS calculations. Drawn lines: $Q$ factor of the $n=1,2,3$ linear groove modes in $d=500 \mathrm{~nm}, \alpha=10^{\circ}$ grooves. For the $n=1$ mode, these $Q$ values correspond to propagation lengths in the range 1-7 $\mu \mathrm{m}$. Vertical dashed lines indicate the energy at which the mode crosses the light line. Squares indicate $m=1$ resonances, triangles indicate $m=2$ ring resonances. Ring radii in this figure were varied for each data point to achieve the required resonance and range from 56 to $522 \mathrm{~nm}$.

\section{Quality factor of ring resonances}

Next we study the quality factor $Q$ of the rings and compare it to the propagation length in linear grooves. In a cavity, the $Q$ factor is a measure of the number of optical cycles that the light remains stored in the cavity. For linear V grooves, an effective $Q$ can be related to the propagation length. We derive it from the LDOS calculations made to find the dispersion relation (as in Fig. 2), deriving $Q$ from the width of each LDOS maximum in the energy domain $(Q$ $=E / \Delta E)$. The drawn curves in Fig. 4 show these $Q$ factors for the three lowest-order $(n=1,2,3)$ modes of a $d$ $=500 \mathrm{~nm}, \alpha=10^{\circ}$ groove, as in Fig. 2(a). Dashed lines indicate the energy at which the mode crosses the light line. For all three modes $Q$ initially increases for increasing energy. This is attributed to the vanishing radiation damping as the plasmon becomes more confined and obtains a $k_{z}$ that is progressively increasing beyond that of free-space light or planar surface plasmons, reducing the radiation loss to those channels. $Q$ then reaches a maximum and decreases for even higher energy. This is due to increased ohmic damping at higher energy. The differences in $Q$ between the different order modes are attributed to differences in dispersion and the corresponding differences in overlap of the mode with the metal.

In a ring resonator, $Q$ can be determined from the width of the resonant LDOS peak as in the spectra of Fig. 3. These data are indicated by the squares $(m=1)$ and triangles $(m$ $=2$ ) in Fig. 4. Each data point corresponds to a different ring, with a radius such that it sustains an $m=1$ or $m=2$ resonance at that energy. The $Q$ values for the ring resonances show a similar increasing and then decreasing trend with energy as the linear groove but at a lower absolute value in all cases. This is due to the fact that ring resonances have radiation damping as an extra loss channel. The difference in $Q$ between linear grooves and ring resonances is less for the higher-order azimuthal $(m=2)$ modes. Such modes correspond do not have a dipole moment and therefore couple less to radiation.

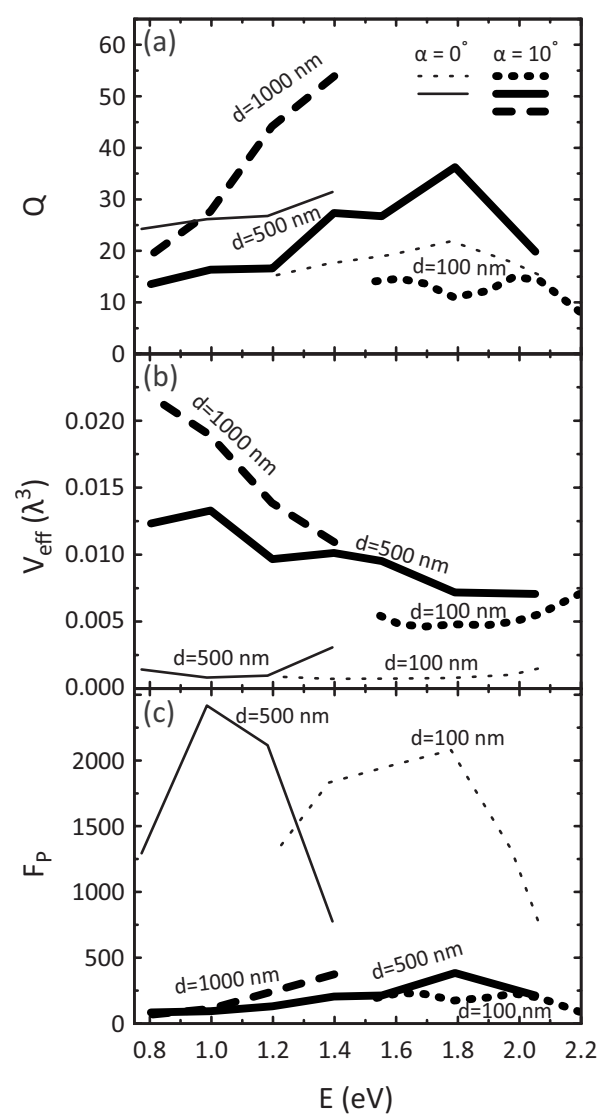

FIG. 5. $Q$ factors, mode volumes and Purcell factors for the $n=1, m=1$ resonance of ring resonators with $d$ $=100,500,1000 \mathrm{~nm}, \alpha=10^{\circ}$ (thick lines), and $\alpha=0^{\circ}$ (thin lines). (a) $Q$ factor. For a large range of geometries, $Q$ remains between 10 and 50. (b) Mode volumes. All mode volumes are far below the diffraction limit $\left(0.125 \lambda^{3}\right)$. Ring resonators with $\alpha=0^{\circ}(10 \mathrm{~nm}$ groove width) show the smallest volumes. For a $d=100 \mathrm{~nm}$ groove at $1.38 \mathrm{eV}$, the mode volume is as small as $0.00073 \lambda_{0}^{3}$. (c) Purcell factors. In the lower three curves $\left(\alpha=10^{\circ}\right)$, a Purcell enhancement of more than 350 is found. For the resonators based on thin grooves $\left(\alpha=0^{\circ}\right)$ Purcell factors reach to over 2000.

Figure 5(a) shows a systematic comparison of $Q$ values for a range of ring geometries. $Q$ factors are plotted for the $m=1$ resonance resulting from the lowest-order groove mode $(n=1)$. Plotted are results from rings with $\alpha=10^{\circ}$ and $d$ $=100,500,1000 \mathrm{~nm}$ (thick lines). Also plotted are results for $\alpha=0^{\circ}, d=100,500 \mathrm{~nm}$ (thin lines). The figure shows that $Q$ factors in these rings with such different shapes are almost all between 10 and 50 (corresponding to peak widths of $\sim 50-100 \mathrm{meV}$ ). Given that the differences in groove shape span a large range for these calculations, it appears that optimization of $Q / V$ in ring resonators is more a matter of mode volume rather than quality factor.

\section{Mode volume of ring resonances}

The effective volume of a resonant mode can be determined by performing the following integral: 


$$
V_{\mathrm{eff}}\left(\mathbf{r}_{e}, \omega\right)=\frac{\int_{V} W(\mathbf{r}, \omega) d^{3} \mathbf{r}}{W\left(\mathbf{r}_{e}, \omega\right)},
$$

where $W$ is the electromagnetic energy density ${ }^{33}$ given by $W(\mathbf{r}, \omega)=\frac{\epsilon_{0}}{2}\left(n^{2}+\frac{2 \omega n \kappa}{\Gamma}\right)|\mathbf{E}(\mathbf{r}, \omega)|^{2}$ using an oscillator model with damping frequency $\Gamma$ for the dielectric constants. $\mathbf{r}_{e}$ is the position of the emitter for which the Purcell factor is to be found. The electric field $E(\mathbf{r}, \omega)$ of a resonant mode is found using BEM by calculating the induced field of a dipole radiating at the resonance energy. In this calculation, the field of the dipole itself is subtracted from the total field. The position $\mathbf{r}_{e}$ of the emitter is chosen to be in the center of a groove, at a depth for which the field is highest.

Figure 5(b) shows the calculated mode volumes for the ring resonances of the same groove geometries as in Fig. 5(a). Each curve represents properties of different rings, with ring radii chosen such that there is a lowest-order $m=1$ resonance at that particular energy. The general trend of decreasing mode volume with energy is attributed to an increase in mode index with energy, which means that relatively smaller rings are needed to obtain a resonance. The increase in effective mode volume for higher energies, as is observed in the $\alpha=0^{\circ}$ and $\alpha=10^{\circ}, d=100 \mathrm{~nm}$ geometries, is not attributed to an increase in the absolute size of the modal field but solely to the decrease in the wavelength to which the volumes are normalized. The figure shows that the mode volumes of rings with $\alpha=10^{\circ}$ follow a similar general trend. This is consistent with the fact that the field of the lowestorder groove mode is localized to the groove bottom and thus not very sensitive to the groove depth. The termination of some of the curves at low energies is caused by cutoff of the groove mode. For higher energies, the size of deeper grooves poses a geometrical limit to the smallest possible ring radius. The smallest mode volume is reached for the $\alpha$ $=0^{\circ}$ ring resonators, with values below $0.001 \lambda_{0}^{3}$. In the highenergy spectral range, this is achieved for $d=100 \mathrm{~nm}$ grooves; for lower energies, deeper grooves are required to reach similar low-mode volume. The smallest mode volume is observed for the (10 nm width), $d=100 \mathrm{~nm}$ ring at 1.38 $\mathrm{eV}$. The V-groove structures with the same width and depth all show higher mode volume, indicating that lowest mode volumes are observed for closely spaced parallel sidewalls. The extremely small mode volume found here is $\sim 180 \times$ smaller than the diffraction limit.

\section{E. Purcell factor of ring resonances}

With a known $Q$ and $V$, the Purcell enhancement can now be calculated from Eq. (1). The index of the medium is taken to be $n=1$, as the field is mostly present in the void. Figure 5(c) shows the Purcell factors for the different ring geometries in Figs. 5(a) and 5(b). For the rings with $\alpha=10^{\circ}$, Purcell factors range to over 350 at $E=1.4 \mathrm{eV}$ for $d$ $=1000 \mathrm{~nm}$ and $1.8 \mathrm{eV}$ for $d=500 \mathrm{~nm}$. Indeed, such a high Purcell factor can be achieved over the broad spectral range from $1.4-1.8 \mathrm{eV}$ by choosing the proper depth. The $\alpha=0^{\circ}$ (10 nm wide) geometries have Purcell factors that reach well beyond 2000 at $1.0 \mathrm{eV}$ for $d=500 \mathrm{~nm}$ and $1.8 \mathrm{eV}$ for $d$
$=100 \mathrm{~nm}$. For these narrow grooves, Purcell factors beyond 2000 can be achieved over a very broad bandwidth of 1.0 $1.8 \mathrm{eV}$ by tuning the ring cavity depth.

From the data in Fig. 5 it follows that the cavity quality factor varies by only a factor $5(Q=10-50)$ while the mode volume varies by a factor 30 . As a consequence, the variations in the Purcell factor are governed by variations in mode volume rather than quality factor.

It is well known that dipole emitters spaced closely to a metal surface experience an enhanced decay rate as a result of nonresonant coupling to a continuum of surface modes. ${ }^{34}$ For example, an emitter spaced at 5 $\mathrm{nm}$ from a planar $\mathrm{Au}$ surface experiences a decay rate that is enhanced approximately 25 times at $1.6 \mathrm{eV}$ [http://www.amolf.nl/download/dipolecalc/], ${ }^{35}$ mainly as a result of coupling to the so-called lossy surface waves. This effect was not taken into account in the calculations presented here. However, in the structures presented here, the nonresonant coupling is substantially smaller than the Purcell enhancement due to coupling to cavity plasmons. As a result, these nonresonant effects can be neglected.

The groove-based resonator geometry presented here is a versatile cavity that allows for large spontaneous emission enhancements over a broad spectral range, enabling experiments with a broad range of emitters (QDs, dyes, rare earth ions) incorporated in its circular void. Recent work ${ }^{27}$ has shown that narrow $(<20 \mathrm{~nm})$ grooves with depth well beyond $100 \mathrm{~nm}$ can be fabricated using focused-ion-beam milling, which brings ultrahigh spontaneous emission enhancements within reach. Further optimization of the geometry should lead to Purcell factors even higher than those reported in the present paper.

\section{CONCLUSIONS}

In conclusion, we have shown that the resonances of plasmonic V-groove ring resonators directly follow from the dispersion in linear V-groove plasmon waveguides. This allows for tuning of the ring cavity resonances by the ring radius. For these resonances, we have calculated the quality factor $Q$ and mode volume $V$ for different groove shapes and ring radii. Quality factors range from 10-50 depending on geometry; mode volumes vary by a factor 30 . Smaller cavities suffer from larger radiation losses. For higher energies, cavity modes are well confined to the bottom of the groove, thus $Q$ and $V$ are less sensitive to depth. The smallest mode volume was found to be $0.00073 \lambda_{0}^{3}$ for a ring based on a $\alpha=0^{\circ}, 100-\mathrm{nm}$ deep, 10 -nm wide groove with a radius of $90 \mathrm{~nm}$. In this plasmon resonator, light can be confined more than 1000 times stronger than in a photonic crystal cavity. A very high Purcell factor, $F_{P}>2000$, can be achieved over a broad range of energies $(1.0-1.8 \mathrm{eV})$ by choosing the proper ring geometry. For a given geometry, similar Purcell enhancement is observed over a broad spectral range $(\sim 50-100 \mathrm{meV})$ due to the moderate cavity $Q$.

This allows for control of spontaneous emission and lightmatter interaction in general, ${ }^{36}$ over broad spectral range, paving the way for plasmon enhanced lasers and broadband light sources. 


\section{ACKNOWLEDGMENTS}

This work is part of the research program "Microscopy and modification of nanostructures with focused electron and ion beams" (MMN) of the "Stichting voor Fundamenteel
Onderzoek der Materie" (FOM), which is financially supported by the "Nederlandse organisatie voor Wetenschappelijk Onderzoek" (NWO). The MMN program is cofinanced by FEI Co. SARA, Amsterdam, is acknowledged for computing services. *vesseur@amolf.nl

${ }^{1}$ K. H. Drexhage, J. Lumin. 1-2, 693 (1970).

${ }^{2}$ E. M. Purcell, Phys. Rev. 69, 681 (1946), Abstract B10.

${ }^{3}$ M. L. Gorodetsky, A. A. Savchenkov, and V. S. Ilchenko, Opt. Lett. 21, 453 (1996).

${ }^{4}$ D. W. Vernooy, V. S. Ilchenko, H. Mabuchi, E. W. Streed, and H. J. Kimble, Opt. Lett. 23, 247 (1998).

${ }^{5}$ J. M. Gérard, B. Sermage, B. Gayral, B. Legrand, E. Costard, and V. Thierry-Mieg, Phys. Rev. Lett. 81, 1110 (1998).

${ }^{6}$ M. Pelton, J. Vukovic, G. S. Solomon, A. Scherer, and Y. Yamamoto, IEEE J. Quantum Electron. 38, 170 (2002).

${ }^{7}$ D. K. Armani, T. J. Kippenberg, S. M. Spillane, and K. J. Vahala, Nature (London) 421, 925 (2003).

${ }^{8}$ B. Gayral, J. M. Gérard, A. Lemaître, C. Dupuis, L. Manin, and J. L. Pelouard, Appl. Phys. Lett. 75, 1908 (1999).

${ }^{9}$ D. Englund, D. Fattal, E. Waks, G. Solomon, B. Zhang, T. Nakaoka, Y. Arakawa, Y. Yamamoto, and J. Vučković, Phys. Rev. Lett. 95, 013904 (2005).

${ }^{10}$ P. Velha, E. Picard, T. Charvolin, E. Hadji, J. Rodier, P. Lalanne, and D. Peyrade, Opt. Express 15, 16090 (2007).

${ }^{11}$ H. Altug, D. Englund, and J. Vuckovic, Nat. Phys. 2, 484 (2006).

${ }^{12}$ S. Noda, M. Fujita, and T. Asano, Nat. Photonics 1, 449 (2007).

${ }^{13}$ Y. C. Jun, R. D. Kekatpure, J. S. White, and M. L. Brongersma, Phys. Rev. B 78, 153111 (2008).

${ }^{14}$ A. Hryciw, Y. C. Jun, and M. L. Brongersma, Opt. Express 17, 185 (2009).

${ }^{15}$ H. T. Miyazaki and Y. Kurokawa, Phys. Rev. Lett. 96, 097401 (2006).

${ }^{16}$ Y. Gong and J. Vučković, Appl. Phys. Lett. 90, 033113 (2007).

${ }^{17}$ M. Kuttge, E. J. R. Vesseur, and A. Polman, Appl. Phys. Lett. 94, 183104 (2009).

${ }^{18}$ M. Kuttge, F. J. García de Abajo, and A. Polman, Nano Lett. 10, 1537 (2010)
${ }^{19}$ S. I. Bozhevolnyi, V. S. Volkov, E. Devaux, and T. W. Ebbesen, Phys. Rev. Lett. 95, 046802 (2005).

${ }^{20}$ I. V. Novikov and A. A. Maradudin, Phys. Rev. B 66, 035403 (2002).

${ }^{21}$ S. I. Bozhevolnyi, V. S. Volkov, E. Devaux, J.-Y. Laluet, and T. W. Ebbesen, Nature (London) 440, 508 (2006).

${ }^{22}$ E. Moreno, S. G. Rodrigo, S. I. Bozhevolnyi, L. Martín-Moreno, and F. J. García-Vidal, Phys. Rev. Lett. 100, 023901 (2008).

${ }^{23}$ E. J. R. Vesseur, F. J. García de Abajo, and A. Polman, Nano Lett. 9, 3147 (2009).

${ }^{24}$ F. J. García de Abajo and A. Howie, Phys. Rev. Lett. 80, 5180 (1998).

${ }^{25}$ F. J. García de Abajo, Phys. Rev. B 59, 3095 (1999).

${ }^{26}$ P. B. Johnson and R. W. Christy, Phys. Rev. B 6, 4370 (1972).

${ }^{27}$ R. de Waele, S. P. Burgos, A. Polman, and H. A. Atwater, Nano Lett. 9, 2832 (2009).

${ }^{28} \mathrm{R}$. de Waele (unpublished).

${ }^{29}$ The LDOS for linear grooves is separated into the contribution of different $k_{z}$ components. This quantity is defined in the $2 \mathrm{D}$ plane normal to the groove direction and it is normalized such that its integral over $k_{z}$ gives the LDOS at a point in 3D space.

${ }^{30}$ J. A. Dionne, L. A. Sweatlock, H. A. Atwater, and A. Polman, Phys. Rev. B 72, 075405 (2005).

${ }^{31}$ R. J. Walters, R. V. A. van Loon, I. Brunets, J. Schmitz, and A. Polman, Nature Mater. 9, 21 (2010).

${ }^{32}$ S. I. Bozhevolnyi, Opt. Express 14, 9467 (2006).

${ }^{33}$ R. Ruppin, Phys. Lett. A 299, 309 (2002).

${ }^{34}$ R. R. Chance, A. Prock, and R. Silbey, Adv. Chem. Phys. 37, 1 (1978).

${ }^{35}$ http://www.amolf.nl/download/dipolecalc/; J. Kalkman, H. Gersen, L. Kuipers, and A. Polman, Phys. Rev. B 73, 075317 (2006).

${ }^{36}$ T. J. Kippenberg, A. L. Tchebotareva, J. Kalkman, A. Polman, and K. J. Vahala, Phys. Rev. Lett. 103, 027406 (2009). 